\title{
Reaction-Diffusion Systems: Front Propagation and Spatial Structures
}

\author{
Massimo Cencini ${ }^{1}$, Cristobal Lopez ${ }^{2}$, and Davide Vergni ${ }^{3}$ \\ 1 INFM Center for Statistical Mechanics and Complexity, Dipartimento di Fisica \\ di Roma "La Sapienza", P.zzle Aldo Moro, 2 Rome, Italy, 00185, \\ Massimo.Cencini@roma1.infn.it \\ 2 Instituto Mediterraneo de Estudios Avanzados (IMEDEA) CSIC-UIB, Campus \\ Universitat Illes Balears Palma de Mallorca, Spain, 07122, \\ clopez@imedea.uib.es \\ 3 Istituto Applicazioni del Calcolo, IAC-CNR V.le del Policlinico, 137 Rome, \\ Italy, 00161, Davide.Vergni@roma1.infn.it
}

\begin{abstract}
After the pioneering works of Kolmogorov, Petrovskii and Piskunov [1] and Fisher [2] in 1937 on the nonlinear diffusion equation and its traveling wave solutions, scientists from many different disciplines have been captivated by questions about structure, formation and dynamics of patterns in reactive media. Combustion, spreading of epidemics, diffusive transport of chemicals in cells and population dynamics are just a few examples bearing witness of the influence of those works in different areas of modern science.
\end{abstract}

\section{Introduction}

In many natural phenomena we encounter propagating fronts separating different phases. An unfortunately familiar example is the front separating burnt from unburnt trees in forest fires. Similarly, propagating fronts play an important role in the speed of epidemics, in population dynamics, or in the propagation of flames and chemical reactions. Most of these, at first glance disparate phenomena find their common denominator in the presence of diffusion (allowing the agent of an epidemic or a chemical substance to spread), and reaction (that is the specific way in which different phases or chemical components react); they are generically referred to as reaction diffusion (RD) systems.

The prototypical model for RD systems is the nonlinear diffusion equation

$$
\frac{\partial}{\partial t} \theta(x, t)=D \frac{\partial^{2}}{\partial x^{2}} \theta(x, t)+F(\theta),
$$

introduced $^{1}$ in 1937 in the seminal contributions of R.A. Fisher [2] and A.N. Kolmogorov, together with I.G. Petrovskii and N.S. Piskunov [1] (hereafter referred to as FKPP), as a model to describe the spreading of an advantageous gene. (1) describes the spatio-temporal evolution of a population

\footnotetext{
${ }^{1}$ As mentioned in Murray (see p. 277 in [3]), (1) was already introduced in 1906 by Luther.

M. Cencini, C. Lopez, and D. Vergni, Reaction-Diffusion Systems: Front Propagation and Spatial Structures, Lect. Notes Phys. 636, 187-210 (2003)

http://www.springerlink. com/

(C) Springer-Verlag Berlin Heidelberg 2003
} 
(concentration), $\theta(x, t)$, of individuals which diffuse with diffusion coefficient $D$, and grow according to a specific rule $F(\theta)$. In FKPP it was shown that (1) admits uniformly translating solutions - traveling waves.

Similar types of propagation phenomena are ubiquitous in Nature. The concepts and mathematical tools developed in $[1,2]$ stand at the foundation of a still increasing number of applications of the reaction diffusion equations in biology, chemistry and physics (see [3-6] and references therein).

The present knowledge on reaction diffusion systems is so vast that it cannot be presented here in a comprehensive and systematic way. Therefore, our discussion will be limited to introductory material. The first part of this chapter is devoted to (1) in one spatial dimension, providing the reader with the main concepts and simplest mathematical tools necessary to understand its behavior. In the second part we enlarge the discussion to generalizations of (1) in moving media with more than one reacting species and, to dimension higher than one.

\section{Front Propagation in the Nonlinear Diffusion Equation}

Perhaps the best way to start our discussion on (1) is to motivate it as originally proposed in FKPP. Consider an area populated by individuals of the same species. Suppose that $\theta(x, t)(\in[0,1])$ is the concentration of the subset of these individuals which possess a particular genotype that makes them favored in the struggle for survival. In particular, assume that the survival probability of individuals with that character is $1+\alpha(\alpha>0)$ times larger than that of individuals without it. Then the evolution of the concentration $\theta$ is ruled out by the standard logistic growth model

$$
\frac{\mathrm{d} \theta}{\mathrm{d} t}=F(\theta)=\alpha \theta(1-\theta) .
$$

The above equation implies that starting from $\theta \approx 0$ there is an initial exponential growth $\theta \sim \exp (\alpha t)$ followed by a saturation at $\theta=1$ due to nonlinearities. Hence $\theta=0$ is an unstable state and $\theta=1$ a stable one.

If, during one generation (the period between birth and reproduction), individuals move randomly in any direction, the concentration evolution is given by (1) with $F(\theta)$ as in (2).

Now if the concentration of individuals with the advantageous genotype is initially different from zero only in a small region, it is natural to ask how it will spread over the space. Specifically, following Kolmogorov et al., let us assume that at $t=0$ there is a localized region in which the density is different from 0 and 1 , and on the left of this region $\theta=1$ while on the right $\theta=0$. By means of the combined effect of diffusion and reaction, the region of density close to 1 will expand, moving from left to right. In other words, 
at long times, $\theta(x, t)$ can be expressed as

$$
\theta(x, t)=\Theta_{v}(x-v t)
$$

meaning that the concentration behaves as a wave with propagation velocity $v$ and shape $\Theta_{v}$.

The problem is to find the limiting shape of the density profile and the limiting rate of its motion. Nowadays, after the efforts of many scientists who extended and generalized Kolmogorov results to different classes of nonlinear terms and generic initial conditions, this problem is well understood (see [5,7-9] and references therein). In the following, we present the modern understanding of it, trying to remain at an intuitive level of discussion.

First of all let us consider the general equation (1), rewritten here for convenience

$$
\frac{\partial}{\partial t} \theta(x, t)=D \frac{\partial^{2}}{\partial x^{2}} \theta(x, t)+F[\theta(x, t)] .
$$

Without specifying the shape of $F(\theta)$, we assume two steady states, an unstable one $(\theta=0)$ and a stable one $(\theta=1)$, i.e. $F(\theta)$ satisfies the conditions

$$
\begin{aligned}
& F(0)=F(1)=0 ; \\
& F(\theta)>0 \text { if } 0<\theta<1 .
\end{aligned}
$$

\section{Pulled versus Pushed Fronts}

Within the assumptions (5), we can distinguish two classes of nonlinear terms. The first one, often indicated as FKPP-like, is characterized by having the maximum slope of $F(\theta)$ for $\theta=0$ (as for the logistic growth model (2), see Fig. 1a). This is the case of the so-called pulled fronts, for which the front dynamics can be understood by linear analysis since it is essentially
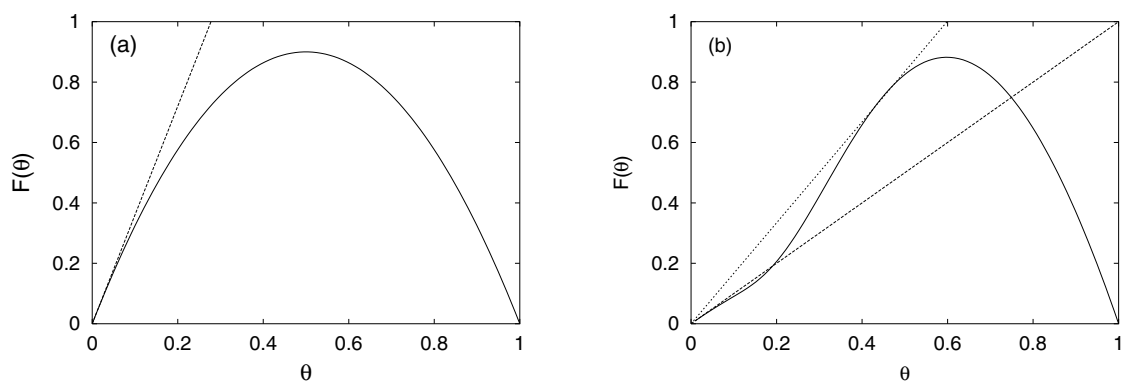

Fig. 1. a A typical FKPP-like production term (pulled dynamics). b A production term which produces a pushed dynamics. The dashed and the dotted straight lines display the linear behaviors $F^{\prime}(0) \cdot \theta$ and $\left.\left(\sup _{\vartheta}\{F(\vartheta) / \vartheta)\right\}\right) \cdot \theta$, respectively. See text for explanation 


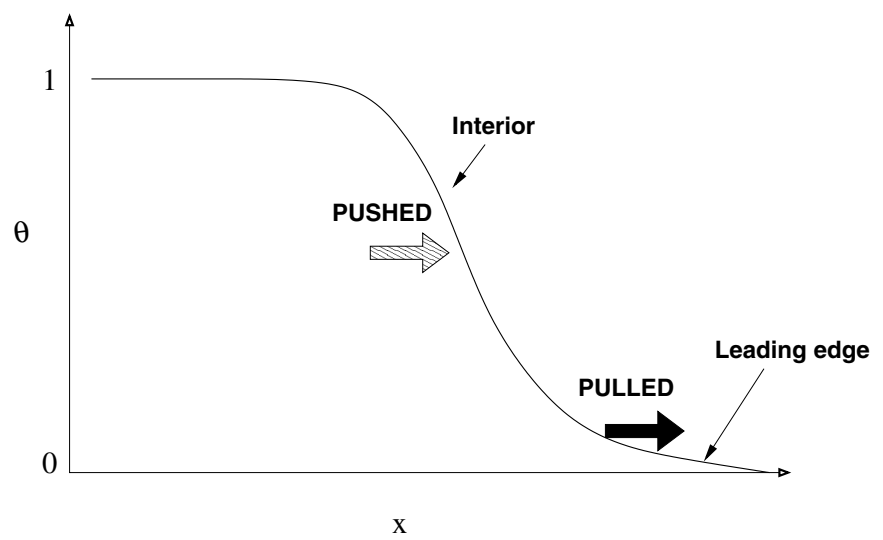

Fig. 2. Pictorial representation of FKPP (pulled) and non FKPP (pushed) fronts

determined by the $\theta(x, t) \approx 0$ region (so the front is pulled by its leading edge, see Fig. 2). In the second class, non FKPP-like, the maximal growth rate is not realized at $\theta=0$ but at some finite value of $\theta$, see Fig. $1 \mathrm{~b}$, where the details of the nonlinearity of $F(\theta)$ are important. In this case front dynamics is often referred as pushed, meaning that the front is pushed by its (nonlinear) interior, Fig. 2. In contrast with the previous case, a detailed non linear analysis of (4) is now required to determine the front speed.

In both pushed and pulled fronts there exists a one parameter family of traveling wave solutions, $\Theta_{v}$, characterized by their velocity, $v$. In their work of 1937, Kolmogorov et al. proved that not all velocities are allowed for pulled fronts. Indeed the following inequality has to be satisfied

$$
v \geq v_{0}=2 \sqrt{D F^{\prime}(0)} .
$$

Furthermore, the equality $v=v_{0}$ is always realized for localized initial conditions as the one mentioned above. This result was lately generalized by Aronson and Weinberger [7] to generic production terms $F(\theta)$. They showed that the minimal allowed front speed, $v_{m i n}$, is bounded by (see Fig. 1)

$$
2 \sqrt{D F^{\prime}(0)} \leq v_{\min }<2 \sqrt{D \sup _{\theta}\left\{\frac{F(\theta)}{\theta}\right\}} .
$$

Note that $F(\theta) / \theta$ is a measure of the growth rate, and that for FKPP dyna$\operatorname{mics}_{\sup _{\theta}}\{F(\theta) / \theta\}=F^{\prime}(0)$ which implies Kolmogorov's result $v_{\text {min }}=v_{0}$.

Equation (6) bounds the minimal front speed but, in principle, front solutions with $v \geq v_{\min }$ are allowed. Therefore, it is important to determine the velocity that is actually selected for a given initial condition. This is the so-called velocity selection problem. 
FKPP-Like Reaction Terms

Here, like Kolmogorov et al., we assume that $F(\theta)$ fulfills the conditions (5) supplemented by

$$
F^{\prime}(0)>0 \text { and } F^{\prime}(\theta)<F^{\prime}(0) \text { for all } 0<\theta \leq 1,
$$

ensuring that $F^{\prime}(0) \equiv \sup _{\theta}\{F(\theta) / \theta\}$. Note that assumptions (5) and (7) are quite reasonable in biological problems and in some chemical reactions. Then from (4) by choosing the frame of reference moving with the front, i.e. with the variable change $z=x-v t$, one obtains the equation for the limiting front profile

$$
D \frac{\mathrm{d}^{2}}{\mathrm{~d} z^{2}} \Theta_{v}(z)+v \frac{\mathrm{d}}{\mathrm{d} z} \Theta_{v}(z)+F\left(\Theta_{v}\right)=0
$$

with boundary conditions $\Theta_{v}(-\infty)=1$ and $\Theta_{v}(+\infty)=0$. In the case of localized initial conditions, Kolmogorov and coworkers rigorously demonstrated, using a very interesting constructive proof, that (8) has a positive definite ${ }^{2}$ solution with speed

$$
v_{0}=2 \sqrt{D F^{\prime}(0)}
$$

Such a solution exists and is unique, apart from a linear transformation $x^{\prime}=$ $x+c$ which does not modify the front profile.

The fact that many solutions with different velocities appear and that $v_{0}$ is the minimal one can be inferred by linearizing (4) around $\theta=0$ (which is the important region in the pulled regime)

$$
\frac{\partial}{\partial t} \theta(x, t)=D \frac{\partial^{2}}{\partial x^{2}} \theta(x, t)+F^{\prime}(0) \theta .
$$

In the neighborhood of $\theta(x, t) \approx 0$, i.e. in the leading edge region, it is reasonable to expect an exponential profile (this is actually always observed) so that for $x \rightarrow \infty$, while $t$ is large but finite, one can write

$$
\theta(x, t) \sim e^{-a(x-v t)},
$$

where $1 / a$ is a measure of the flatness/steepness of the profile. Substituting the last expression in (10) one finds the link between asymptotic front shape and speed,

$$
v=D a+\frac{F^{\prime}(0)}{a}
$$

\footnotetext{
${ }^{2}$ Notice that if the initial concentration is non negative, $\theta \geq 0$, it will remain so under the dynamics (4). This follows immediately by interpreting (4) as the heat equation with heat source $F(\theta)$, which by $(5)$ is never negative.
} 
which is the so-called dispersion relation. This equation implies that profiles with different velocities are allowed and that there exists a minimal velocity, $v_{\text {min }}$, realized for

$$
a^{*}=\sqrt{\frac{F^{\prime}(0)}{D}}
$$

corresponding to $v_{\min }=2 \sqrt{F^{\prime}(0) D}$, which is Kolmogorov's result (9).

It is possible to show that front solutions with $a>a^{*}$ are unstable [8-10]. This is a crucial point which is at the core of the speed selection problem. In fact, one observes that for steep enough initial conditions $\left(a \geq a^{*}\right)$, the front always relaxes to the one of minimal speed. On the other hand, if the initial conditions are sufficiently flat, $a<a^{*}$, the front propagates with a rate given by (12), thus the broader the front $(a \rightarrow 0)$ the faster it moves. We will comment further on the selection mechanism at the end of this section.

Let us now show how, for localized initial conditions, the front always converges to the minimal speed solution [10]. Writing $\theta(x, t)=\exp \left[F^{\prime}(0) t\right] \phi(x, t)$, (10) reduces to the heat equation for the new variable $\phi\left(\partial_{t} \phi=D \partial_{x}^{2} \phi\right)$ which can be easily solved. In terms of $\theta$ the solution reads

$$
\theta(x, t)=\exp \left(F^{\prime}(0) t\right) \int_{-\infty}^{\infty} \mathrm{d} y \theta(y, 0) \frac{\exp \left[-\frac{(x-y)^{2}}{4 D t}\right]}{\sqrt{4 \pi D t}} .
$$

Now introducing the coordinate $z=x-v_{0} t$ with $v_{0}$ given by (9), and assuming that $\theta(y, 0)$ is different from 0 only in a small region (say around the origin) one obtains

$$
\theta(x, t)=\Theta_{v}(z) \propto \frac{\exp \left(-z \sqrt{F^{\prime}(0) / D}-z^{2} / 4 D t\right)}{\sqrt{t}} .
$$

This equation tells us that the front shape asymptotically approaches an exponential profile with steepness $\xi \propto 1 / a^{*}=\sqrt{D / F^{\prime}(0)}$, and that the front speed $v_{0}$ is reached as

$$
v(t)-v_{0} \propto \frac{1}{t},
$$

i.e. in an algebraically slow way ${ }^{3}$.

\section{Pushed Fronts}

For pushed fronts, conditions (7) are not satisfied. This implies that the maximal growth rate is not realized at $\theta=0$ but in the non linear interior of the

\footnotetext{
${ }^{3}$ Actually it has been shown that the prefactor $\frac{1}{t}$ is universal in FKPP-like fronts [10]. Here we just stress that the algebraic convergence comes out from the $1 / \sqrt{t}$ prefactor characteristic of the Gaussian propagator.
} 
front (see Figs. 1b and 2). As a relevant example, we mention thermally activated chemical reactions (such as combustion) which are typically modeled using the Arrhenius production term,

$$
F(\theta)=(1-\theta) e^{-\theta_{c} / \theta},
$$

where below the activation concentration, $\theta_{c}$, essentially no reaction takes place.

Contrary to pulled fronts, where the front speed can be determined by linear analysis, a fully nonlinear treatment is required here. However, there still exists a minimal velocity $v_{\min }$ below which no solutions are allowed. The point is that now $v_{\min }>v_{0}$, with $v_{0}$ given by (9).

A simple way to understand how a minimal velocity larger than $v_{0}$ appears can be found in [9]; here we report the main idea. We have seen that the leading edge is always exponential, so that for large $z=x-v t$

$$
\Theta_{v}(z)=A_{v}^{F} \exp \left[-a_{F}(v) z\right]+A_{v}^{S} \exp \left[-a_{S}(v) z\right]
$$

where $a_{F}(v)$ and $a_{S}(v)\left(>a_{F}(v)\right)$ are the flat and the steep modes, respectively. In the above analysis (see (11)) to derive the dispersion relation (12), we considered only the flat decreasing mode, because asymptotically it is the leading one. However, seeing that (8) is a second order equation, a superposition like (18) is expected in general. The constants $A_{v}^{F}$ and $A_{v}^{S}$ depend on the velocity $v$, and on the nonlinear part of the front through matching conditions with the interior. As before, allowed front solutions should be positive, meaning that at least $A_{v}^{F}$ should be positive. For large enough $v$, front solutions are allowed since both $A_{v}^{S}$ and $A_{v}^{F}$ are positive. If, for $v=v_{\text {min }}$, the amplitude of the leading mode $A_{v}^{F}$ goes to zero, then, for continuity, $A_{v}^{F}$ will become negative for $v<v_{\text {min }}$, by continuity. As a consequence, the corresponding $\Theta_{v}$ is not an allowed solution. Precisely at $v=v_{\min }$ (18) reduces to the single fast exponential decrease. Note that for pulled fronts at $v=v_{0}$, $a_{S}\left(v_{0}\right)=a_{F}\left(v_{0}\right)$; see [10] for a discussion about this point.

Also in this case, depending on the flatness/steepness of the initial profile, the asymptotic front speed may be larger than $v_{\min }$ or may relax to the minimal one.

\section{Velocity Selection}

For initial conditions steep enough (including localized initial conditions), the front dynamics is always attracted by the minimal speed solution which, for pulled fronts, corresponds to the linear prediction (9) and in general satisfies the bounds (6). The detailed proof of this statement requires a non trivial analysis which depends crucially on the simplicity of the model under consideration. However, while remaining at the level of a general discussion, it is interesting here to briefly recall the ideas at the basis of the modern way in which the speed selection problem is understood. 
The crucial concept is that of stability in the moving frame or marginal stability condition $[5,8,9]$, which may be formulated as follows. If one adds a small perturbation to the leading edge of a moving front, then the front is stable if it outruns the perturbation (it is left behind and readsorbed) and unstable if the perturbation persists at long times. The natural front is selfsustained, i.e., the growth of the perturbation from the unstable to the stable state should be the cause of front propagation. Therefore, the selected front should be the one which is marginally stable with respect to the perturbation. In this framework, the Aronson and Weinberger result [7] is equivalent to the statement that the front with the lowest velocity is marginally stable with respect to local perturbations of the state $\theta=0$. This criterion can be generalized also to the case of pushed fronts $[8,9]$.

\subsection{Multiple Steady States}

Up to now, we have considered reaction terms having only two steady states. However, in a broad class of problems in nonlinear chemistry and population dynamics, such as enzymatic reactions or insects spreading [3], multiple steady states may be present, meaning that the production term have $N \geq 3$ zeros in $[0,1]$

$$
F\left(\theta_{i}\right)=0, \quad \text { for } \quad i=1, \ldots, N .
$$

These fixed points can be stable or unstable and more complicated propagation phenomena can appear.

In order to provide the reader with some basic ideas, let us introduce a simple and instructive description of the front propagation problem exploiting an analogy with the dynamics of a point particle $[5,8]$. To make it evident let us rewrite (8) as

$$
D \ddot{y}+v \dot{y}+F(y)=0,
$$

where $y \equiv \Theta_{v}$ and the dots indicate derivatives with respect to the variable $z=x-v t$, which here represents time. The reader will recognize that this is the equation for a classical particle moving in a potential

$$
V(y)=\int^{y} \mathrm{~d} y^{\prime} F\left(y^{\prime}\right),
$$

and damped with a friction coefficient $v$.

By using this analogy, the existence of a minimal velocity below which no uniformly translating fronts exist has a clear interpretation [10]. Let us reconsider, for the sake of simplicity, the case of pulled fronts in the framework of linear analysis (10). We assume a parabolic potential $V(y)=-F^{\prime}(0) y^{2} / 2$. Due to the friction term, at sufficiently long times, an exponential decay, $y(t) \sim \exp (-a t)$ is expected (i.e. an exponential front profile at large distances). Substituting this behaviour in (19) one obtains that

$$
a(v)=\frac{v \pm \sqrt{v^{2}-4 F^{\prime}(0) D}}{2 D} .
$$



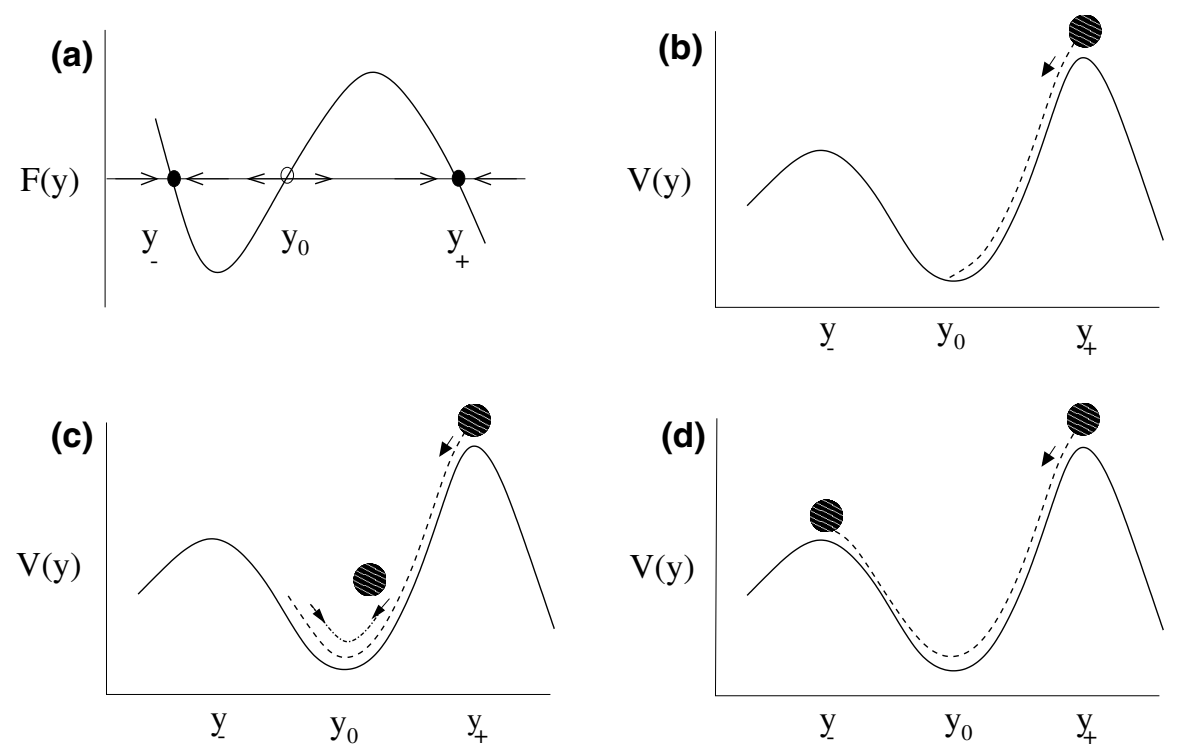

Fig. 3. Pictorial representation of the particle in the potential analogy. a Generic production term with three steady states, two stable (filled circles) and one unstable (empty circle). b,c,d The potential obtained with (20), see text for explanation on the different situations

Now if the damping is not strong enough $\left(v<2 \sqrt{D F^{\prime}(0)}\right)$ the particle will reach $y=0$ in a finite time implying that the front becomes negative, which is not allowed. Therefore, $v=2 \sqrt{D F^{\prime}(0)}$, is the minimal friction ensuring that the particle will asymptotically reach $y=0$, and so the front remains positive and monotonic.

This analogy becomes very useful in the presence of many steady states. For example, let us consider a generic function $F(y)$ having three zeros [5] (see Fig. 3a): an unstable state at $y_{0}$ and two stable states at $y_{ \pm}$, corresponding to the minimum and the two maxima of the potential $V(y)$, respectively. A front (in general any structure connecting two different states) is a trajectory connecting one of the maxima with the minimum, e.g. $y_{+}$with $y_{0}$.

For the parabolic potential previously examined, for large enough $v$ (say $v \geq v_{1}$ ), the damping is efficient and $y_{0}$ is reached at $t \rightarrow \infty$, i.e. the front is monotonic (see Fig. 3b). Below the critical damping $v_{1}$, there is an overshoot before reaching $y_{0}$ and the particle will pass $y_{0}$ going uphill toward $y_{-}$before ending in $y_{0}$. Below another critical value $v_{2}<v_{1}$, the approach to the minimum may be underdamped the particle oscillate for ever in the valley (Fig. 3c); that is, the leading edge of the front is oscillatory. There also exists a critical value $v_{3}\left(<v_{2}<v_{1}\right)$ for which the particle lands precisely at $y_{-}$, the front joins two stable states (Fig. 3d). For $v<v_{\text {min }}$ the orbit goes to $-\infty$, which does not represent a finite solution of (19). Notice that contrary 
to fronts propagating from an unstable state to a stable one, for those joining two stable states there exists a unique solution and not a family of solutions - only one speed value is allowed.

\section{Reaction Diffusion Systems in Physics, Chemistry, and Biology}

In the previous sections we have examined the one-dimensional reaction diffusion equation. Now, after a brief overview on the wide range of applicability of this equation in different areas of science, we will focus on some specific issues such as multicomponent chemical reactions, combustion and an ecological problem concerning the distribution of plankton in the ocean.

\section{Chemical Reactions}

The most natural application of the nonlinear diffusion equation is the study of chemical reactions taking place in the environment or in living organisms. In multicomponent chemical reactions, one considers generalizations of (1) where many species with their interrelations and diffusion constants are present. Moreover, depending on the media where the reaction takes place, one can have either an advection term (reaction in fluid flows), or spatial a dependence in the diffusion coefficient [11] (reaction in heterogeneous media). In the presence of many species, the problem becomes much more difficult. Indeed a large range of behaviors, from oscillations to chaos [12], can be found. As it will become clear in Sect. 3.1, this gives rise to much more complex spatio-temporal propagation phenomena than in the simple one-dimensional case (see $[3,4,13]$ and references therein).

\section{Combustion Theory}

Among the many chemical reactions, for its theoretical and practical importance, we mention the problem of combustion [14], which has been the first application [15] of concepts and tools originally introduced in FKPP. Combustion problems are complicated not only by the presence of many reactants, but also by the fact that the burning of combustible takes place in a moving medium, usually a fluid. Hence one has to include in (1) the advection by the fluid velocity, and we speak about reaction advection diffusion systems. This increases enormously the complexity and difficulty of the problem because the fluid motion is usually very complex due to turbulence [16], which is another fundamental aspect of Kolmogorov's interests (see Chaps. 7 and 8). In Sec. 3.2, we will discuss in details this problem.

\section{Population Dynamics and Ecology}

The contributions of Fisher and Kolmogorov on the reaction diffusion equation had a prominent role in the development of mathematical tools in population dynamics and ecology (see, e.g., [3,6]). Indeed (1) and its generalizations 
are at the basis of many studies ranging from the rate of advance of invading species [3] to human genetics and expansion [17]. Closely related to these works, and building upon them, has been the development of models to explain patchiness in the distribution of organisms [6], which is an important issue in the study of plankton and zooplankton in oceans [18]. In Sec. 3.2 we will provide the reader with a brief overview on this ongoing research area.

\section{Pattern Formation and Developmental Biology}

Finally, it is important to mention that RD systems do not only give rise to propagation phenomena but also to standing-patterns. Steady heterogeneous spatial structures, or patterns, appear in nature running from the very small scales, like in colonies of bacteria, to astronomical ones, like the spiral structure of some galaxies [3,5]. The interest is then in understanding pattern formation.

A central role in pattern formation studies was played by another great scientist, namely A. Turing ${ }^{4}$ who, in a classic paper [19], showed that pattern forming instabilities may arise when considering RD mechanisms. Even if there is no room here to properly treat this problem, we mention that already from the early work of Turing it was realized the potentiality of RD modeling for developmental biology [3]. Probably the most striking example in this context is offered by morphogenesis, i.e., the development of patterns and forms from the initially homogeneous mass of cells in the embryo of many animals. For instance, think of the richness and beauty of patterns in animal coats or butterflies leaves [3].

Nowadays, many different formation mechanisms have been identified, and pattern formation in RD systems is a very vast and important area in the study of non-equilibrium physical and chemical systems [5].

\subsection{Multi-components Reaction Diffusion Systems}

The general mathematical expression for a multicomponent reaction diffusion system is just an extension of (4) to an $N$-components vector field $\boldsymbol{\theta}=$ $\left(\theta_{1}, \ldots, \theta_{N}\right)$

$$
\frac{\partial \theta_{i}}{\partial t}=D_{i} \nabla^{2} \theta_{i}+F_{i}\left(\theta_{1}, \ldots, \theta_{N}\right)
$$

where $F_{i}\left(\theta_{1}, \ldots, \theta_{N}\right)$ is the reaction term for the $i$-th species, and $D_{i}$ its diffusivity. Very complex behaviors appear now depending on the intrinsic reaction dynamics given by the $F_{i}$ 's. To illustrate the phenomenology of these systems, we will use two paradigmatic examples: the celebrated BelusovZhabotinskii (BZ) chemical reaction [12], and the predator-prey (PP) systems [6], with many applications in populations dynamics and ecology.

\footnotetext{
${ }^{4}$ From a historical point of view it is interesting to know that Turing was not aware of the works of Kolmogorov, Petrovskii and Piskunov [1], which was poorly known in the West for many years.
} 
The BZ reaction is probably the most widely studied, both theoretically and experimentally, oscillating (it can also have excitable or chaotic behavior) chemical reaction. It involves more than 40 elementary reactions which result in changes of several dozens of intermediate substances. The basic mechanism consists of the oxidation of malonic acid by bromate ions, catalyzed by, e.g., ferroin and ferriin. For some values of the reagent concentrations, periodic oscillations are observed in these catalysts, producing a periodic color change oscillating between red (ferroin) and blue (ferriin). More details can be found, for example, in [12]. Concerning the PP systems, the simplest model involves two components, predators and preys (the concentrations of which are denoted by $v$ and $u$, respectively), and can be written

$$
\begin{aligned}
& \frac{d u}{d t}=r u\left(1-\frac{u}{u_{0}}\right)-c v f(u)+D \nabla^{2} u, \\
& \frac{d v}{d t}=a v f(u)-b v+D \nabla^{2} v,
\end{aligned}
$$

where $r, c, a, b, u_{0}$ are positive parameters and $D$ is the diffusivity. The first term on the rhs of (22) indicates the intrinsic birth-death of the preys; in the second term, $f$ is the prey consumption function per predator. Analogously, the first term on the rhs of (23) is the benefit from predation and the second one models predators' death.

It is important to remark on the universality of behavior in this class of systems: similar wave patterns to those found in the BZ reaction (see below Figs. 4 and 7) or the PP model appear also in many other reaction diffusion systems having the same dynamical behavior.

Let us start with the case in which the $F_{i}$ 's give rise to an oscillatory dynamics, inducing a periodic evolution of the $\Theta_{i}$ fields in time. A front may develop, for example, if the oscillation of any part of the system is perturbed, and traveling wave trains move through the system. In the context of predator-prey systems, these periodic traveling waves can be originated by the invasion of a predator population into the prey population. In one dimension, wave train solutions are of the form

$$
\theta_{i}(x, t)=\Theta_{i}(\omega t-k x),
$$

where $\omega$ is the frequency, $k$ the wavenumber, and $\Theta_{i}$ is a periodic function of the phase. Therefore the advancing front leaves behind it a spatially periodic pattern. In two spatial dimensions, these wave trains are concentric circles, referred to as target patterns (see Fig. 4 for an example appearing in the BZ reaction).

Other interesting behaviors appear in excitable systems, which are characterized by the presence of activator and inhibitor components. The activator has a catalytic effect both on itself (autocatalysis) and on the inhibitor which, in turn, depletes the activator production. At the end, the system has a stable fixed point as the only attractor for the dynamics. Examples of excitable systems may be found in semiconductor lasers with optical feedback [20], neural 


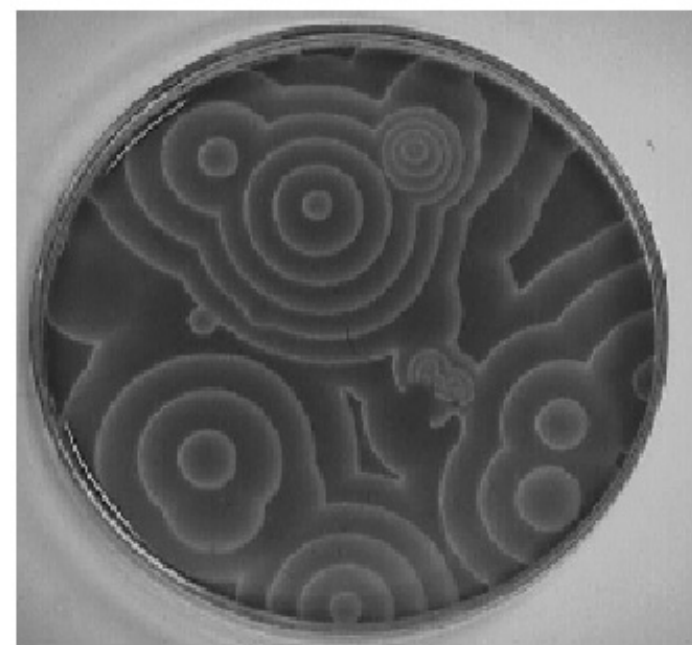

Fig. 4. Target patterns for the BZ reaction

communications [3], and populations dynamics [3]. Moreover, for some values of the parameters, the $\mathrm{BZ}$ reaction and $\mathrm{PP}$ models also behave as excitable systems.

The main feature of this kind of systems is the way in which they respond to perturbations. Typically there exists a threshold value such that if the perturbation goes above it the system reaches the fixed point only after a long excursion in the phase space. This behavior usually appears when the activator has a temporal response much faster than the inhibitor, so that it takes some time before stopping the growth of the activator. The threshold property is characteristic of cubic nonlinearities in the reaction term, as exemplified by the Fitzhugh-Nagumo (FN) equations,

$$
\begin{aligned}
& \frac{\partial u}{\partial t}=u(a-u)(u-1)-v+D \frac{\partial^{2} u}{\partial x^{2}}, \\
& \frac{\partial v}{\partial t}=b u-\gamma v,
\end{aligned}
$$

originally introduced as a mathematical model for neural activity [21], where $u$ is the activator, $v$ the inhibitor, and $a, b, \gamma$ are positive parameters.

The threshold property can be understood by studying the nullclines of the system (Fig. 5), which are obtained by equating to zero the rhs of (25) with $D=0$. If the value of $u$ is smaller than the threshold $a$, $u$ quickly returns to the origin (the stable fixed point) and the spatial perturbation dies out. On the contrary, if the perturbation is larger than the threshold, the fixed point is reached after a large excursion in both $u$ and $v$ passing through the points $0 B C D 0$. 


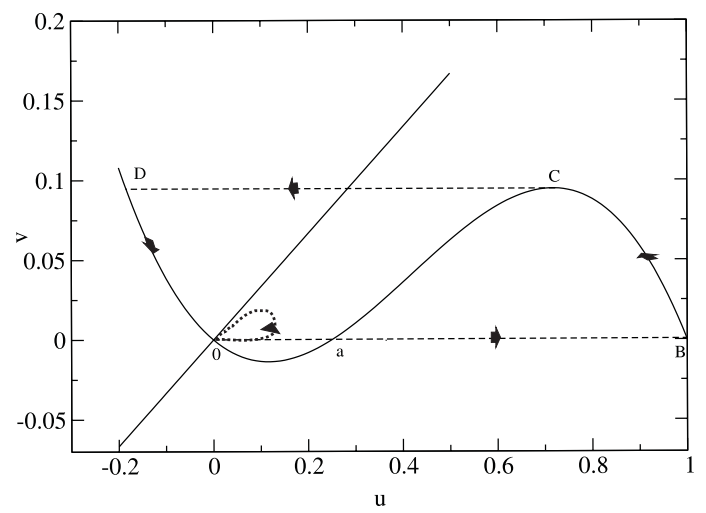

Fig. 5. Phase trajectories for $u$ and $v$ depending on whether the perturbation is larger than or smaller than the threshold $a$. Solid lines are the nullclines

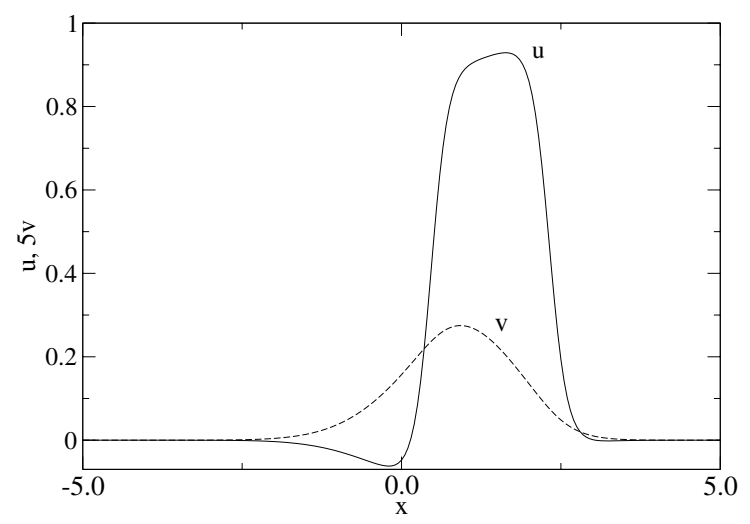

Fig. 6. Pulse-like front solution for a one-dimensional excitable medium

The propagation of the excitation through neighboring points, coupled diffusively, generates traveling pulses as the one shown in Fig. 6. In two dimensions we have circular propagating waves. Pulse waves have been shown to exist in generic excitable models, but the values of the propagation velocity and the shape of the pulse depend on the specific reaction term. In particular for the FN system, one can show [3] that, in the limit of small $b$ and $\gamma$, the wave speed is given by $c=\sqrt{D / 2}(1-2 a)$. In two dimensions, when a propagating pulse is broken at a point, it begins to rotate around the ends, producing spiral waves (see Fig. 7 for a typical example in the BZ reaction). There are also many other relevant occurrences of spiral waves in natural systems. Just to name a few, let us mention fibrillating hearts, where small regions contract independently and the spreading through the cortex of damaged brains [3]. 


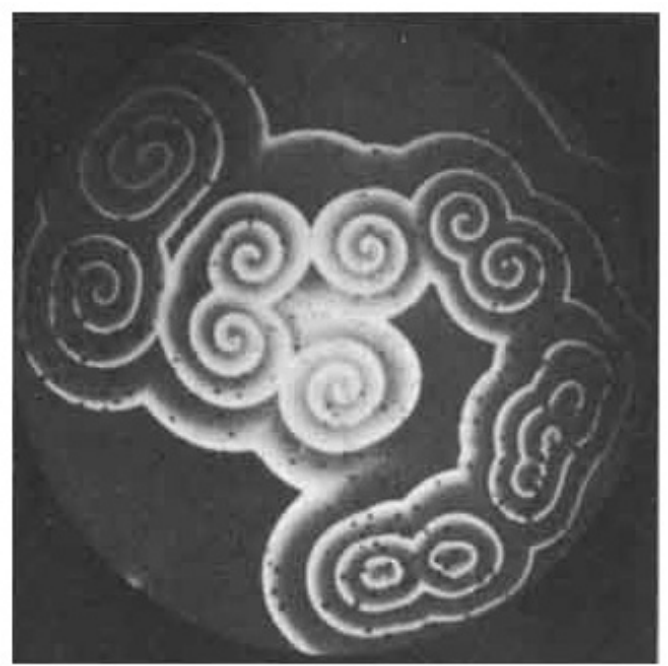

Fig. 7. Spiral waves observed in the BZ reaction

However, the phenomenology can be much more complicated. For example, target patterns can also be formed in an extended excitable medium if the pulses are emitted periodically, and spiral waves can be formed by breaking target waves by stirring the medium, or by noise-induced effects.

Let us now briefly comment the case of chaotic reaction dynamics (see $[4,5]$ for more details). An interesting case, widely observed in predator-prey systems, appears when periodic wave trains become highly disordered, loosing their periodicity. In this case, very irregular spatial patterns appear behind the front. Moreover, spiral waves may become highly disordered and organize themselves in chaotic sets that continuously form and decay (the so-called transition to spatio-temporal chaos).

\subsection{Advection Reaction Diffusion Systems}

Reaction diffusion processes taking place in moving media such as fluids are of considerable importance, e.g. in combustion, atmospheric chemistry, and ecological problems. As we saw in Chap. 8, even passive scalars, substances which are simply transported by the flow, display a very complex behavior both in laminar and turbulent flows. When reaction is taken into account the problem becomes even more complex. In fact the heat release associated with chemical reactions will affect the velocity field, and transport is not passive any more. However, even when the feedback of the advected scalar on the moving medium can be neglected (like in certain aqueous autocatalytic reactions), the dynamics of the reacting species is strongly complicated by the presence of the velocity field. 
Similarly to passive scalars, one can adopt two complementary points of view. A first possibility is to consider particles modeling reagents (or individuals, in ecological problems), which move under the effect of a velocity field and thermal noise (diffusion), and reacting when they come into contact this is the Lagrangian viewpoint (see also Chap. 8). Alternatively, adopting an Eulerian viewpoint (see also Chap. 7), one considers a field of concentration which evolves according to the advection reaction diffusion (ARD) equation, which for one species reads

$$
\frac{\partial \theta}{\partial t}+\mathbf{u} \cdot \nabla \theta=D \nabla^{2} \theta+F(\theta),
$$

where $\mathbf{u}$ is the velocity field; we wrote (26) for an incompressible flow $(\boldsymbol{\nabla} \cdot \boldsymbol{u}=$ 0 ) for simplicity. In the most general formulation of the problem, one also has to consider the Navier-Stokes equation for $\mathbf{u}$ with a term accounting for the feedback of $\theta$ on the velocity field. The two points of view can be related through an elegant mathematical formulation in terms of the Feynman-Kac formula [22].

\section{Chemical Processes in Fluid Flows}

Among the many chemical reactions that take place in fluids, one of the most important is combustion [14]. The general problem is very difficult due to the presence of many components which react in a complicated way and which modify the flow via heat release, thus enhancing the complexity of the flow generating turbulence. Turbulence itself plays an important role in increasing the mixing of reagents (see Chap. 8) and therefore improving the efficiency of combustion processes [16]. For example, in the spark-ignition engine, fuel and oxidizer are firstly mixed by turbulence before the spark ignites the mixture.

For a complete mathematical formulation of the general problem, one has to consider $N$ reacting species $\theta_{i}$ which evolve according to (26)

$$
\frac{\partial \theta_{i}}{\partial t}+\mathbf{u} \cdot \nabla \theta_{i}=D_{i} \nabla^{2} \theta_{i}+F_{i}\left(\theta_{1}, \ldots, \theta_{N}, T\right),
$$

with their own diffusivity constant, $D_{i}$, and reaction kinetics, $F_{i}$, that depends on the temperature, $T$. The temperature itself is transported by the flow and modifies it through buoyancy, so that the Navier-Stokes equation for the velocity field with back-reaction should be considered. Usually the dependence of $F_{i}$ on the temperature is of the Arrhenius type (17) [15]. It is obvious that this set of equations is too complicated for us to give a satisfactory non-technical treatment of the problem.

However, some new phenomena which arise because of the presence of the advecting velocity field can be appreciated, even considering a single reacting species and neglecting the back-reaction on the fluid, i.e. remaining at the level of the ARD equation (26). For the sake of simplicity, let us consider 

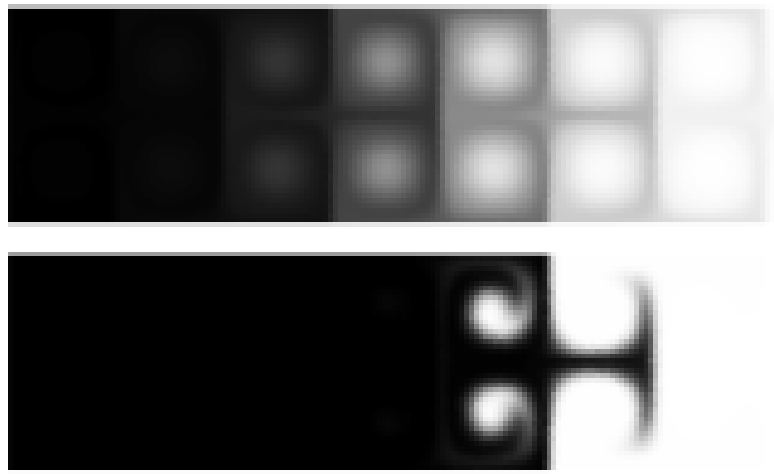

Fig. 8. Front propagation in a pipe-like geometry in two-dimension. The boundary conditions are $\theta(-\infty, y)=1$ and $\theta(+\infty, y)=0$. The grey scale indicates a concentration going from fresh material $(\theta=0$, white) to burnt material $(\theta=1$, black). The advecting velocity field is a cellular flow, $u_{x}=U \sin x \cos y$ and $u_{y}=-U \sin y \cos x$, where $U$ is the stirring intensity. The upper image is for low $D a$, i.e. slow chemistry; note the thickness of the reaction zone, $\xi$, which extends over many velocity characteristic length scales, $L$ (here the transversal length). The lower image is for high $D a$, fast reaction, here $\xi \ll L$

(26) in a pipe-like geometry with a given velocity field (see Fig. 8). For our illustrative purpose, the velocity field is left unspecified. We only assume that it is characterized by its intensity, $U$ (the root mean square velocity) and its typical length scale, $L$ (the correlation length, or in the pipe-problem the transverse length). In addition, let us assume that $F(\theta)$ is of the FKPP-type with $\theta=0$ and $\theta=1$ representing, the unstable (unburnt) and stable (burnt) states, respectively. Let us rewrite $F(\theta)=f(\theta) / \tau$ where $\tau$ is the typical temporal scale of the chemical kinetics, and $f$ is the rescaled production term with $f^{\prime}(0)=1$. Suppose that at the initial time on the left there is burnt material and the rest of the pipe is filled with fresh unburnt reagent. This can be seen as an extremely simplified burning process or chemical reaction. As time advances the front separating burnt from unburnt material will advance from left to right with a speed $v_{f}$. Now the question is how the front speed and shape will be modified by the presence of the velocity field, $\mathbf{u}$.

In a fluid at rest, $\mathbf{u}=0$, we saw that the propagation speed is given by $v_{0}=2 \sqrt{D / \tau}(9)$, the laminar front speed in combustion jargon. Moreover, the thickness of the region where the reaction takes place, $\xi$, is roughly given by $\xi \sim \sqrt{D \tau}$ (see (13)). In a moving fluid it is natural to expect that the front will propagate with an average (turbulent) speed $v_{f}$ greater than $v_{0}$. The turbulent front speed $v_{f}$ will be the result of the interplay among the flow characteristics, $L$ and $U$, the diffusivity $D$, and the chemical time scale $\tau$. The analysis can be simplified by introducing two non-dimensional num- 
bers: the Damköhler number $D a=L /(U \tau)$, the ratio of advective to reactive time scales, and the Peclet number $P e=U L / D$, the ratio of diffusive to advective time scales. Usually one is interested in the limit of high $P e$ number, when advection is stronger than diffusion. The front speed is expected to be expressed as $v_{f}=v_{0} \phi(D a, P e)$ which is in general larger than $v_{0}$ [22].

The study of the detailed dependence of $v_{f}$ on $D a$ and $P e, \phi(D a, P e)$, is non-trivial. However, some limiting cases can be identified. A particularly simple case is when the reaction is very slow, $D a \ll 1$. In this regime the thickness of the reaction zone is much larger than the typical velocity length scale, $\xi \gg L$ (see Fig. 8). On length scales larger than $L$ the transport properties of an advected scalar (or of particles in the Lagrangian viewpoint) are known to be well described by an effective diffusion constant, $D_{\text {eff }}$, usually much larger than the molecular diffusivity, $D$ (see [23] and references therein). As a consequence, the reaction zone behaves as if the diffusion coefficient is $D_{\text {eff } f}$. In other words, on scales much larger than $L(26)$ reduces to (1) with $D \rightarrow D_{\text {eff }}$. So that the theory discussed in Sect. 2 applies $[22,24]$ with

$$
v_{f} \approx 2 \sqrt{D_{e f f} / \tau}
$$

Apart from slow biological reactions, or when the stirring by the velocity field is extremely intense, most of reactions of interest have time scales comparable or faster than the advection time, $L / U$ (fast reaction). Therefore the previous result cannot be applied. However, it is interesting to note that the rhs of (28) is a rigorous upper bound to the turbulent front speed $v_{f}$ [22]. A possible approach in the case of fast reactions is to renormalize both the diffusion constant and the chemical time scales. But while the computation of the renormalized diffusion coefficient is based on powerful, well-established mathematical methods [23], the renormalization of $\tau$ can only be approached phenomenologically [22].

Another limit is when $D, \tau \rightarrow 0$, while remaining the ratio $D / \tau$ constant; here, the reaction zone thickness $\xi \sim \sqrt{D \tau}$ shrinks to zero, while the laminar front speed $v_{0}$ stays finite). In this case, the ARD equation reduces to the so-called $G$-equation [16]

$$
\frac{\partial G}{\partial t}+\mathbf{u} \cdot \nabla G=v_{0}|\nabla G|
$$

The iso-scalar surface (line in two dimension), say $G=0$, represents the front position. Equation (29) has a simple geometrical interpretation: in the absence of stirring $(\mathbf{u}=\mathbf{0})$ the front evolves according to the Huygens principle, i.e., a point $\mathbf{x}$ belonging to the front moves with a velocity $\mathbf{v}(\mathbf{x})=v_{0} \hat{\mathbf{n}}(\mathbf{x})$, $\hat{\mathbf{n}}(\mathbf{x})$ being the perpendicular direction to the front surface in $\mathbf{x}$. The effect of the velocity field is to wrinkle the front, increasing its area and thereby its speed [16]. Indeed the front speed in this limit is linked to the amount of material which is burnt per unit time, which increases as the front area increases. Assuming a velocity field with a turbulent spectrum, Yakhot [25] 
proposed that at large flow intensities $\left(U \gg v_{0}\right) V_{f} \propto U / \sqrt{\ln U}$. We do not know whether this prediction is correct or not, although the fact that $v_{f}$ has an almost linear behavior with $U$ (here corrected by $\sqrt{\ln U}$ ) seems to be a generic feature in laboratory and numerical experiments up to moderately high intensities.

\section{Plankton Patchiness}

The large importance of plankton distributions in the sea must not be underestimated. They are at the lowest level of the ocean food chain, and among the most important ingredients for understanding the interchange of $\mathrm{CO}_{2}$ between the atmosphere and the oceans and, consequently, the phenomenon of global warming [26].

A characteristic that is well known since the earliest in situ observations, recently verified by satellite remote sensing and detailed numerical simulations [27], is plankton patchiness, i.e., the inhomogeneity of plankton spatial distributions. These analyses identify filaments, irregular patches, sharp gradients, and other complex structures involving a wide range of spatial scales in the concentration patterns, which typically extend from medium scales $(\sim 10 \mathrm{~km})$ to very large ones $(\sim 1000 \mathrm{~km})$, associated with the major ocean currents and gyres (Fig. 9).

Traditionally, patchiness has been variously attributed to the interplay of diffusion and biological growth, oceanic turbulence, diffusive Turing-like instabilities, and nutrient or biological inhomogeneities [28]. Advection by unsteady fluid flows and predator-prey interactions are only recently emerging as two key ingredients able to reproduce the main qualitative features of plankton patchiness [18]. Therefore, the proper mathematical framework for this problem is that of advection reaction diffusion systems with many components (27).

The reaction term usually takes into account three different trophic levels and their interactions: nutrients $(N)$, phytoplankton $(P)$ and zooplankton $(Z)$. The nutrients are inorganic materials dissolved in water that can be assimilated by the phytoplankton organisms; the zooplankton grazes on the latter. The interactions among $N, P$ and $Z$ are schematically sketched in Fig. 10. As one can see they are of the predator-prey type with competition for resources and mortality. Moreover, the uptake of nutrients by phytoplankton, and the grazing of these by the zooplankton are also taken into account. Regarding the advection by oceanic flows, the mechanism which is now emerging as a key feature in explaining the observed filament-like structures of the concentration patterns is chaotic advection [29], i.e. the presence of highly chaotic trajectories of the fluid particles even in relatively simple Eulerian flows. In fact the continuous stretching and folding of fluid elements induced by the flow is considered to be one of the basic ingredients for the generation of patchiness, see [27] for a recent review. 


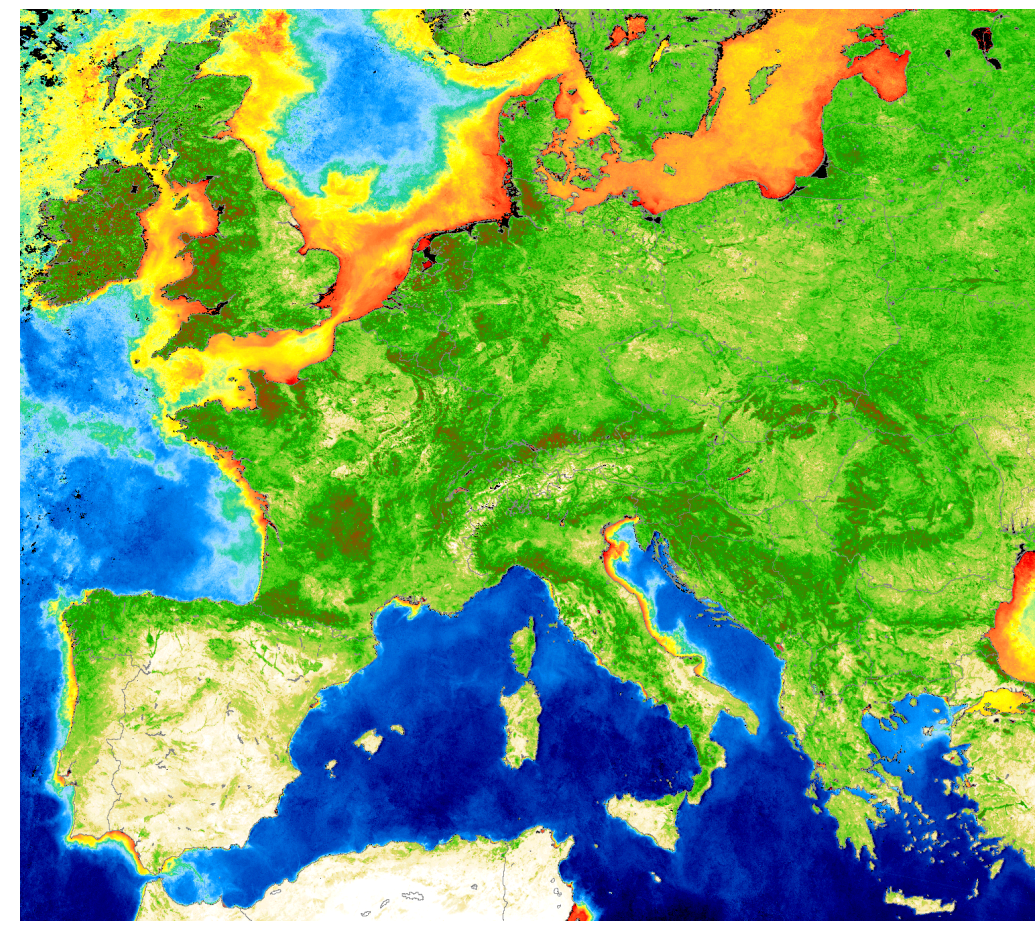

Fig. 9. Satellite image of phytoplankton pigment (chlorophyll) concentration in Western Europe (Courtesy by Marine Environment Unit, image from SeaWiFS Images Archive: http://www.me.sai.jrc.it)

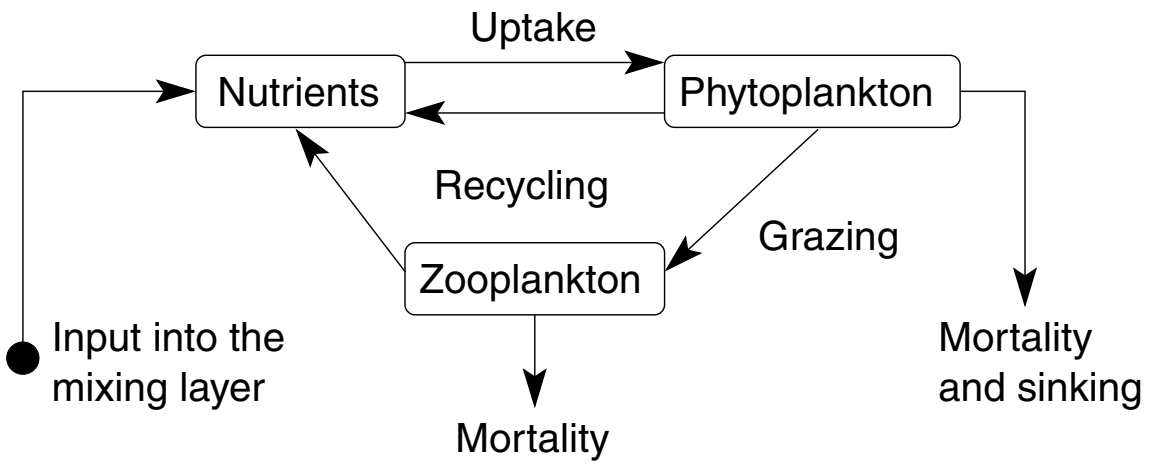

Fig. 10. The processes in the NPZ models 
Here we limit the discussion to a simple model [30] constituted by three trophic levels where, instead of considering explicitly the nutrients, we introduce the carrying capacity, $C$, defined as the maximum phytoplankton content that a parcel of water can support in the absence of grazing. Considering a relaxational dynamics for $C$, and assuming equal diffusivities (which is reasonable because mixing in plankton communities is largely due to seawater turbulence), the model is

$$
\begin{aligned}
& \frac{\partial C}{\partial t}+\mathbf{u} \cdot \nabla C=\alpha\left(C-C_{0}(\mathbf{x})\right)+D \nabla^{2} C \\
& \frac{\partial P}{\partial t}+\mathbf{u} \cdot \nabla P=P(1-P / C)+D \nabla^{2} P \\
& \frac{\partial Z}{\partial t}+\mathbf{u} \cdot \nabla Z=P Z-\delta Z^{2}+D \nabla^{2} Z
\end{aligned}
$$

where $\alpha$ describes the relaxation of $C$ onto an imposed spatially dependent carrying capacity, $C_{0}(\mathbf{x})$ (see [30] for more details), and $\delta$ is the $Z$ mortality. The velocity field $\mathbf{u}(\mathbf{x}, t)$ is incompressible and it is assumed to give rise to chaotic advection, which implies that the separation between two fluid particles, $|\delta \mathbf{x}(t)|$, initially close $(|\delta \mathbf{x}(0)| \ll 1)$ typically diverges in time at a rate given by the Lyapunov exponent of the flow $\lambda_{F}>0$,

$$
|\delta \mathbf{x}(t)| \propto|\delta \mathbf{x}(0)| e^{\lambda_{F} t} .
$$

In the absence of the flow and with $D=0$, the dynamics is attracted by the stable fixed point of (30): $C^{*}=C_{0}(\mathbf{x}), P^{*}=C_{0} \delta /\left(\delta+C_{0}\right)$, and $Z^{*}=P^{*} / \delta$. Thus the chemical Lyapunov exponent ${ }^{5} \lambda_{C}$ is negative. This simple model displays an interesting transition depending on the value of $\lambda_{F}$ and $\lambda_{C}$. If $\left|\lambda_{C}\right|>\lambda_{F}$ the plankton distribution is smooth, while if $\left|\lambda_{C}\right|<\lambda_{F}$, i.e. when the flow is enough chaotic to overcome the stability of plankton dynamics, the asymptotic spatial distribution of plankton has fractal properties.

Another remarkable feature of this model is its ability to reproduce a well-known experimental fact related to the behavior of the power spectrum, $\Gamma(k)$ ( $k$ is the wavenumber), of the species distributions. Specifically, analysis of transects taken by oceanographic ships have shown that the power spectra of zooplankton and phytoplankton have a power law behavior characterized by different scaling exponents [18]: $\Gamma_{P}(k) \propto k^{-\beta_{P}}$ and $\Gamma_{Z}(k) \propto k^{-\beta_{Z}}$, with $\beta_{P} \neq \beta_{Z}$, indicating the different distributions of $P$ and $Z$. Furthermore, $\beta_{P}$ and $\beta_{Z}$ seem to be different from 1, the scaling exponent expected for passive scalars (such as temperature and salinity). Therefore, $P, Z$ and the temperature field are distributed in a very different manner, $Z$ being much more irregularly distributed than $P$, i.e., $1<\beta_{P}<\beta_{Z}[18,30]$. In the model (30) the power spectrum scaling exponents can be computed in terms of

\footnotetext{
${ }^{5}$ That is the Lyapunov exponent of the dynamical systems obtained by (30) with $\mathbf{u}=\mathbf{0}$ and $D=0$.
} 
the Lyapunov exponents $\lambda_{F}$ and $\lambda_{C}$ as $\beta_{P}=\beta_{Z}=1+2\left|\lambda_{C}\right| / \lambda_{F}<1$, which partially reproduces the observations. However, a slightly more sophisticated model has obtained the result $\beta_{P}<\beta_{Z}$ (see article on page 470 of [27]), which is closer to observations.

The complete characterization of plankton patchiness requires the introduction of more refined observables than the power spectrum. For instance, one can define the $q$ th structure functions of, say, phytoplankton, as

$$
S_{q}(\delta r)=\left\langle|P(\mathbf{x}+\delta \mathbf{x}, t)-P(\mathbf{x}, t)|^{q}\right\rangle,
$$

where the bracket represents averaging over locations $\mathbf{x}, \delta r=|\delta \mathbf{x}|$ and $q$ is a positive number. Interestingly, as observed in turbulence and passive scalars (see Chaps. 7, 8), in the limit $\delta r \rightarrow 0$, structure functions have a power law behavior given by $S_{q} \propto \delta r^{\zeta_{q}}$. Moreover, the exponents $\zeta_{q}$ display a non-trivial dependence on $q$, namely they deviate from the linear dimensional estimation $\zeta_{q}=q(1-\beta) / 2$ (where $\beta$ is the power spectrum scaling exponent). These deviations are the signature of the multifractal behavior of plankton distribution. Remarkably, multifractality naturally arises in the framework of models like (30) due to the fluctuations of finite-time Lyapunov exponents (see [30] for a detailed discussion on this point).

The most important lesson one can learn from this simple model is that from the interplay of a smooth flow, which accounts for the physics, and a (simplified) stable interacting dynamics, the biology, one can have a very irregular (multifractal) spatial distribution of population concentrations. Moreover, the relevant quantities describing the inhomogeneities of these distributions, such as the power spectrum or structure function scaling exponents, can be expressed in terms of the Lyapunov exponents that characterize, separately, the dynamics of the flow and of the plankton populations.

\section{References}

1. A.N. Kolmogorov, I. Petrovskii and N. Piskunov, "A study of the diffusion equation with increase in the amount of substance and its application to a biology problem", in Selected works of A.N. Kolmogorov, ed. V.M. Tikhomirov, Vol. I, p. 242, Kluwer Academic Publishers, London (1991); original work Bull. Univ. Moscow, Ser. Int. A, 1, 1 (1937)

2. R.A. Fisher, "The wave of advance of advantageous genes", Ann. Eugenics, 7, 353 (1937)

3. J.D. Murray, Mathematical biology, Springer-Verlag, Berlin (1993)

4. Y. Kuramoto, Chemical Oscillations, Waves, and Turbulence, Springer-Verlag, Berlin (1984)

5. M.C. Cross and P.C. Hohenberg, "Pattern formation outside equilibrium", Rev. Mod. Phys., 65, 851 (1993)

6. A. Okubo and S.A. Levin, Diffusion and ecological problems, Springer-Verlag, Berlin (2001)

7. D.G. Aronson and H.F. Weinberger, "Multidimensional nonlinear diffusion arising in population genetics", Adv. Math., 30, 33 (1978) 
8. E. Ben-Jacob, H.R. Brand, G. Dee, L. Kramer, and J.S. Langer, "Pattern propagation in nonlinear dissipative systems", Physica D, 14, 348 (1985)

9. W. van Saarloos, "Front propagation into unstable states: Marginal stability as a dynamical mechanism for velocity selection", Phys. Rev. A, 37, 211 (1988).; W. van Saarloos, "Front propagation into unstable states. II. Linear versus nonlinear marginal stability and rate of convergence", Phys. Rev. A, 39, 6367 (1989)

10. U. Ebert and W. van Saarloos, "Front propagation into unstable states: universal algebraic convergence towards uniformly translating pulled fronts", Physica D, 146, 1 (2000)

11. J. Xin, "Front propagation in heterogeneous media", SIAM Review, 42, 161 (2000)

12. S.K. Scott,Oscillations, Waves and Chaos in Chemical Kinetics, Oxford University Press, (1994)

13. J. Ross, S.C. Müller, and C. Vidal, "Chemical waves", Science, 240, 460 (1988)

14. F.A. Williams, Combustion Theory, Benjamin-Cummings, Menlo Park (1985)

15. Y.B. Zel'dovich and D.A. Frank-Kamenetskii, "A Theory of thermal propagation of flame", Acta Physicochimica U.R.S.S., Vol. XVII, 1-2, 42 (1938)

16. N. Peters, Turbulent combustion, Cambridge University Press, Cambridge (2000)

17. A.J. Ammerman and L.L. Cavalli-Sforza, The neolithic transition and the genetics of population in Europe, Princeton University Press, Princeton (1984)

18. E.R. Abraham, "The generation of plankton patchiness by turbulent stirring", Nature 391, 577 (1998)

19. A.M. Turing, "The chemical basis of morphogenesis", Phil. Trans. R. Soc. London B, 237, 37 (1952)

20. M. Giudici, C. Green, G. Giacomelli, U. Nespolo, and J. Tredicce, "Andronov bifurcation and excitability in semiconductor lasers with optical feedback", Phys. Rev. E, 55, 6414 (1997)

21. R. Fitzhugh, "Impulses and physiological states in theoretical models of nerve membranes", Biophys. J., 1, 445 (1961). J. Nagumo, S. Arimoto, and S. Yoshizawa, "An active pulse transmission line simulating 1214-nerve axons", Proc. IRL, 50, 2061 (1960)

22. M. Abel, A. Celani, D. Vergni, and A. Vulpiani, "Front propagation in laminar flows", Phys. Rev. E, 64, 046307 (2001)

23. A.J. Majda, and P. R. Kramer, "Simplified models for turbulent diffusion: Theory, numerical modeling and physical phenomena", Phys. Rep., 314, 237 (1999).

24. P. Constantin, A. Kiselev, A. Oberman, and L. Ryzhik, "Bulk Burning Rate in Passive - Reactive Diffusion", Arch. Rational Mechanics, 154, 53 (2000)

25. V. Yakhot, "Propagation velocity of premixed turbulent flame", Combust. Sci. Technol., 60, 191 (1988)

26. P.M. Cox, R.A. Betts, C.D. Jones, S.A. Spall, and I.J. Totterdell, "Acceleration of global warming due to carbon-cycle feedbacks in a coupled climate model", Nature, 408, 184 (2000)

27. Focus Issue on Active Chaotic Flow, Chaos, 12, 372 (2002), editors Z. Toroczkai and T. Tél

28. D.L. Mackas, K.L. Denman, and M.R. Abbott, "Plankton patchiness: biology in the physical vernacular", Bull. Mar. Sci., 37, 652 (1985) 
29. J.M. Ottino The Kinematics of Mixing: Stretching, Chaos and Transport, Cambridge University Press, Cambridge (1989)

30. C.López, Z. Neufeld, E. Hernández-García, and P.H. Haynes, "Chaotic advection of reacting substances: Plankton dynamics on a meandering jet", Phys. Chem. Earth, B 26, 313 (2001) 